\title{
BMJ Open Study protocol: effects, costs and distributional impact of digital primary care for infectious diseases - an observational, registry-based study in Sweden
}

\author{
Jens Wilkens (D) , ${ }^{1}$ Hans Thulesius, ${ }^{1,2}$ Eva Arvidsson, ${ }^{3,4}$ Anna Lindgren, ${ }^{5}$ \\ Bjorn Ekman ${ }^{1}$
}

To cite: Wilkens J, Thulesius $\mathrm{H}$, Arvidsson E, et al. Study protocol: effects, costs and distributional impact of digital primary care for infectious diseases-an observational, registry-based study in Sweden. BMJ Open 2020;10:e038618. doi:10.1136/ bmjopen-2020-038618

\section{- Prepublication history for} this paper is available online. To view these files, please visit the journal online (http://dx.doi. org/10.1136/bmjopen-2020038618).

Received 18 March 2020 Revised 10 June 2020 Accepted 15 July 2020
Check for updates

(C) Author(s) (or their employer(s)) 2020. Re-use permitted under CC BY-NC. No commercial re-use. See rights and permissions. Published by BMJ.

For numbered affiliations see end of article.

Correspondence to Dr Jens Wilkens; jens.wilkens@med.lu.se

\section{ABSTRACT}

Introduction The ability to provide primary care with the help of a digital platform raises both opportunities and risks. While access to primary care improves, overuse of services and medication may occur. The use of digital care technologies is likely to continue to increase and evidence of its effects, costs and distributional impacts is needed to support policy-making. Since 2016, the number of digital primary care consultations for a range of conditions has increased rapidly in Sweden. This research project aims to investigate health system effects of this development. The overall research question is to what extent such care is a cost-effective and equitable alternative to traditional, in-office primary care in the context of a publicly funded health system with universal access. Three specific areas of investigation are identified: clinical effect; cost and distributional impact. This protocol describes the investigative approach of the project in terms of aims, design, materials, methods and expected results.

Methods and analysis The research project adopts a retrospective study design and aims to apply statistical analyses of patient-level register data on key variables from seven regions of Sweden over the years 2017-2018. In addition to data on three common infectious conditions (upper respiratory tract infection; lower urinary tract infection; and skin and soft-tissue infection), information on other healthcare use, socioeconomic status and demography will be collected.

Ethics and dissemination This registry-based study has received ethical approval by the Swedish Ethical Review Authority. Use of data will follow the Swedish legislation and practice with regards to consent. The results will be disseminated both to the research community, healthcare decision makers and to the general public.

\section{BACKGROUND}

As healthcare systems come under increasing fiscal and operational pressures, growing attention is given to the need to strengthen the role of primary care to prevent illness and promote population health. ${ }^{1-3}$ In parallel, expectations are rising among policy-makers

\section{Strengths and limitations of this study}

- This research project applies a system-wide approach by designing studies that cover several outcome areas, recognising that impact from new forms of primary care varies across performance areas.

- The project builds up and will use a database on individual patients and their characteristics that is uniquely large in the Swedish context.

- The studies will not build on random allocation of patients to either of the two models of care, which may negatively affect the strength of conclusions about causal effects.

- The studies will also not be able to differentiate between different forms of digital primary care (eg, synchronous and asynchronous consultations), or an expected future situation where digital care is more integrated with in-office services.

about the use of digital care technologies to enhance the performance of healthcare provision. ${ }^{45}$ From a health systems perspective, there are several potential opportunities and possible risks. Purchasers of services are attracted by potential efficiency gains and savings on healthcare spending. For patients, accessing care by means of a digital platform reduces access barriers in the form of travel and waiting time. At the same time, fears have been raised that easier access to primary care through digital medical service providers may lead to overuse and create inequities in the use of healthcare services. ${ }^{5-7}$

The experience of expanded provision of digital primary care in Sweden over the past 3 years is a case in point. Since 2016, the number of digital primary care consultations has increased 10-fold, reaching around 60 000 consultations per month. ${ }^{8}$ While users 
of digital care generally express satisfaction with this service, policy-makers and experts in general practice have warned of the potential risks of overuse of medical services and medication leading to cost escalations. ${ }^{9}$

As large-scale use of digital primary care in the context of a publicly funded healthcare system is a relatively recent phenomenon, significant gaps in the understanding and evidence of the effects of digital care exist. The purpose of this research protocol is to describe the aims, methods and expected findings of a research project that aims to address some of these gaps.

\section{Aim and objectives of the research project}

The overall aim of this research project is to evaluate the extent to which digital primary care constitute a costeffective and equitable alternative to traditional, in-office primary care in the context of a publicly funded health system. The general approach of the project is to compare the effects of digital care with those of traditional primary care services. Digital care is defined as care provided through digital channels for consultation beyond physical meetings, telephone, mail and email. It includes both synchronous video and asynchronous text and can be provided by both dedicated digital providers and traditional offices. To ensure a balanced and relevant comparison between the two models of primary care, the analyses will focus on three illnesses that are common in both types of care: upper respiratory tract infection (URTI), lower urinary tract infection (LUTI) and skin and soft-tissue infection (SSTI).

The specific objectives of the study are to:

1. Evaluate effects of digital primary care in terms of overall utilisation of primary care services and of compliance with prescription guidelines.

2. Compare the costs of digital primary care with those of in-office primary care for the chosen conditions.

3. Analyse utilisation of digital primary care across socioeconomic and demographic characteristics of patients.

\section{Context of primary care in Sweden}

Healthcare in Sweden is the responsibility of the 21 regions. ${ }^{10}$ The Regions fund medical care by raising income taxes and provide services through publicly owned and managed hospitals and primary care clinics, or by contracting with private providers. The institutional organisation of primary care is characterised by relatively large healthcare clinics that employ several different types of medical professionals. There is a total of some 1140 such clinics in the country, around 2/3 of which are publicly owned. Primary care providers receive the largest share of their reimbursement through a needsadjusted capitation (approximately $82 \%$ of total reimbursement) based on a listing system with free choice of provider. A minor share of revenue comes from a fee-for-service payment. Both parts of reimbursement, and their relative share, vary by region. Adult patients pay a user fee that also varies across Regions; on average around US $\$ 20$ per visit to a primary care physician, with a nationally imposed ceiling amount of approximately US $\$ 110$ per year. ${ }^{11}$

While primary care services are generally regarded as being of high quality, Sweden has a long-standing issue with waiting times to primary care. ${ }^{10} 12$ Indeed, in the most recent review of access to healthcare services, the National Board of Health and Welfare found that no region was able to live up to the national patient guarantee mandate of providing care within 7 days of initial contact. ${ }^{13}$

Access to primary care is particularly poor outside of normal office hours ${ }^{14}$ and have most likely contributed to the relatively rapid uptake of digital primary care services offered by a total of 13 private and public providers. ${ }^{9}$ The two largest providers of digital care in Sweden are KRY and Min Doktor. Together, they provide around three quarters of all digital consultations. These visits are funded by a flat fee per patient contact from the region where the patient is resident, regardless if the patient is listed with a primary care provider or not. A copayment of around US $\$ 10$ is levied on all digital visits. ${ }^{15}$

The entire resident population in Sweden enjoys formal entitlement to both forms of primary care. As private actors continue to expand digital services, the Regions and private providers of traditional in-office primary care also develop digital primary care. While digital care currently only constitutes around $2 \%$ of total primary care visits, it is expected that this share will grow over the coming years. Understanding the implications of this process is regarded as vital for effective policy development and decision-making (with the COVID-19 pandemic, which has caused a large upsurge in demand for digital primary care from March 2020, the increase over time can be expected to grow even faster).

\section{REVIEW OF EXISTING EVIDENCE ON DIGITAL PRIMARY CARE}

The first part of this review includes original research articles published in peer-reviewed journals with a focus on high-income countries. The second part of the review includes systematic reviews of the evidence on digital primary care. Given the comparative novelty of digital primary care, the current evidence base is relatively limited and largely inconclusive as to the effects of digital care on relevant outcomes.

In the Swedish context, Ekman ${ }^{16}$ showed that digital primary care was overall around $40 \%$ less costly than traditional primary care. Half of the cost difference arise on the provider side and half on the patient side. A recent study used a small sample of Swedish patients to investigate if digital primary care reduced visits to traditional primary care clinics in the Skåne region. ${ }^{17}$ The authors found no support for this hypothesis but could not rule out that the higher use of traditional services among those who also used digital care was due to other factors not controlled for in the analysis.

Many studies have shown that low-income population groups consume relatively more primary care than 
high-income groups, as compared with specialist and hospital services, also after controlling for differences in needs. ${ }^{18-20}$ Urban and younger population groups use digital services more frequently, but there is to date no studies available on the distributional effects of digital primary care and how this compares with the traditional form. $^{8}$

In the UK, the users of the recently evaluated Babylon digital primary care application were generally younger than the average primary care patient. The evaluation did not find that use of digital primary care had any impact on the use of secondary care. Importantly, antibiotic prescription rates were lower than the overall rate, possibly due to harder restrictions in prescription rules for this form of service. ${ }^{21}$ Many evaluations of digital services focus on a specific diagnosis or application, and results are not necessarily applicable to primary care services generally. For example, a UK study of Skype consultations for patients with diabetes found the service to be equally safe and effective as in-office visits. ${ }^{22}$

A study with a relatively large sample found similar rates of antibiotic use and guideline-concordant antibiotic management as for traditional care, with the exception of less appropriate streptococcal testing. ${ }^{23}$ Ray et al found that digital care for children generated more antibiotic prescriptions and the patient was less likely to receive correct antibiotic management compared with children visiting traditional care. ${ }^{24}$

Several studies, including those using similar diagnoses as in the current project, have shown that digital care is less costly per visit. One study shows the largest savings in cost per visit occurs for the patient, while provider costs differ less between the two forms of care. ${ }^{25}$ Gordon $e t$ $a l$ showed that digital visits generated a similar level of follow-up visits as in-office visits, but less laboratory tests, resulting in lower costs per episode of care. ${ }^{26}$ Mehrotra $e t$ $a l$ similarly found lower use of laboratory tests, although higher rates of antibiotic prescription. ${ }^{27}$ Even though several studies show digital visits can be provided at lower cost than in-office visits, the introduction of digital services has generated larger volumes of additional visits, resulting in larger overall spending in some settings. ${ }^{28} 29$

\section{Literature reviews}

Armfield et al have reviewed the literature of Skype as a tool for providing digital care in 2012 and 2015. The first review concludes an increasing use of web-based consultations but no evidence for how performance is affected. ${ }^{30}$ The second review found more articles on the use of Skype, but a lack of formal evaluation of its clinical and economic benefits. ${ }^{31}$ A review of reviews of costeffectiveness in eHealth services for somatic conditions found a positive effect in the majority of studies, although few studies of family medicine were available. ${ }^{32}$ A review of economic analyses comparing telehealth services with traditional care found a majority of studies showed lower costs for the telehealth form of provision, although most of these studies only compared provider costs and did not have a patient perspective.$^{33}$ A regional health technology unit in Sweden found no conclusive evidence on clinical benefits and cost effectiveness of digital consultations for emerging symptoms in primary care, as compared with traditional physical visits. ${ }^{34}$ Bashshur et al conclude that evidence on cost effectiveness in favour of digital primary care is still scarce, but growing. ${ }^{35}$ It is noticeable that most literature reviews on the subject of digital care do not target primary care specifically, show mixed results in terms of effects and costs, and find limitations in sample sizes and outcome measures. ${ }^{36}$

\section{METHODS AND ANALYSIS}

A key analytical aim in any evaluation of an intervention or programme is to be able to control for any confounding factor that may affect the outcome measures. One way to do this is to randomise participation into either an intervention group or a control group. ${ }^{37}$ In the current case of digital primary care in Sweden, randomisation of patients to either digital care or traditional care is not possible. As described below, the current study will aim to address these challenges by means of quasi-experimental methods.

\section{Study design and data sources}

This research project applies a retrospective, observational (non-randomised) design, using patient-level register data from seven regions of Sweden for the years 2017-2018. It is expected that the final database will contain data on some 1000000 individuals (around 10\% of the entire population of Sweden). No previous study of digital primary care in Sweden has taken a systemwide approach by designing studies that covers several outcome areas. It is also unique in collecting a large database combining detailed clinical data from several regions in Sweden.

\section{Sample and data collection}

The sample of individuals consists of all patients who were diagnosed with at least one of the three index diagnoses URTI, LUTI, SSTI, in one of the target regions during the study period of 1 January 2017 to 31 December 2018. The seven regions (Jämtland-Härjedalen, Stockholm, Örebro, Östergötland, Kronoberg, Halland and Jönköping) constitute a fair representation of Swedish regions in terms of demographic and socioeconomic characteristics, rural and urban populations, and geographical location.

The data will be collected from four different types of sources: (1) Regions ( $\mathrm{n}=7)$; (2) digital providers $(\mathrm{n}=2)$; (3) National Board of Health and Welfare (three separate databases); and (4) Statistics Sweden (two separate databases). While there is no national database on primary care in Sweden, the existence of a national identification number enables the collection of data from different sources and subsequent linking individuals into a single database. 
Table 1 Variables, indicators and sources of information

$\begin{array}{llll}\text { Variable } & \text { Indicator } & \begin{array}{l}\text { Source of } \\ \text { information }\end{array} & \text { Comment }\end{array}$

\section{A) Identification}

id
Personal identification number

Visit/contact identification number Statistics Sweden, To identify all separate primary care Regions

B) Index diagnoses

i) URTI

ii) LUTI

iii) SSTI

\section{Upper respiratory tract infection Regions}

Lower urinary tract infection

Skin and soft-tissue infection
Statistics Sweden, Regions, Providers
Anonymised data including a nonidentifiable id number will be provided to the researchers

To identify all separate
visits and contacts

Sample variables

ICD (International Classification of Diseases) -10 chapters $B, H, J, R$

ICD-10 chapters N, O

ICD-10 chapters

A, B, F, H, I, K, L, M, N, O, P, T, Z
Regions

Regions

Other diagnoses

i) Diabetes

\section{ii) COPD}

iii) Hypertension

Total number of diagnoses Indicators to control for health status

Diabetes mellitus

Regions ICD-10 chapters E10, E11, E12, E13, E14, E15, E16, E17, E18

Chronic obstructive pulmonary disease

Regions

ICD-10 chapter J44

Hypertension

Regions

ICD-10 chapters I10, I11, I12, I13, I14, I15

Regions

Any other diagnosis registered in patient record

\section{C) Primary care providers}

i) Digital provider

Indicator if digital contact during KRY, Min Doktor study period

\section{ii) Traditional primary care provider \\ Indicator if traditional consultation Regions during study period}

Date and time; category of provider; laboratory examination; medical prescription

Date and time; type of consultation; category of provider; laboratory examination; medical prescription

iii) After-hours visit

Indicator if after-hours primary

Regions

care visit (non-emergency) during

study period

Date and time; category of provider; laboratory examination; medical prescription

\section{D) Other care}

Other types of care

Emergency visits, inpatient care, outpatient care

\section{National Board of}

Health and Welfare

Indicators of other types of care during study period

\section{E) Background variables}

\section{i) Socioeconomic indicators}

$\begin{array}{ll}\text { ii) Sociodemographic } & \begin{array}{l}\text { Age, sex, marital status, country } \\ \text { of birth }\end{array}\end{array}$

After-hours visits refer to visits to clinics during evenings, weekends and nights. As a general starting point, the study will view these visits as part of traditional primary care.

To ensure an effective data collection process and in order to avoid a situation where the researchers need to handle either original data (not anonymised) or an encryption key for the anonymised data, all data will be collected by a special unit of Statistics Sweden. Table 1 describes the indicators and the sources of information.

Data on digital consultations will be collected from the two largest providers of digital primary care, KRY and Min Doktor and from Jönköping Region. Due to the national system for inter-regional billing, the two providers of digital care operated out of this region during the study period. The Jönköping Region keeps a database on these consultations enabling the identification of this group of patients.

Regional health administrations will provide data on diagnoses and procedures from all relevant primary care visits. Each visit contains information on diagnosis, location of provider, type of visit, staff category, time of visit, 
laboratory testing results and prescription of medication. The National Board of Health and Welfare provides data on prescribed and dispensed pharmaceuticals from a Swedish national drug registry and data on specialist visits in outpatient facilities and hospitals are collected from a national Swedish patient registry. Data on income, education, place of birth, residence, marital and labour market status are provided by Statistics Sweden's databases.

The principal investigator of the research project (BE) is responsible for handling the final data set in accordance with national regulations and the principles and guidelines for data handling at Lund University. The storage, management and handling of the data set are described in a special data management plan developed by the research group.

As stated as one of the limitations of the study, this registry-based quantitative approach will not address important questions such as the perceptions of medical staff and patients of digital primary care. The design has however been developed by a multiprofessional research team including practising specialists in family medicine.

\section{Patient and public involvement}

The research project is developed in response to a new form of providing primary care services and an emerging change in patient care seeking behaviour. The design of the studies has however not involved patients or the general public. The project should be complemented by research on patients' experiences and preferences.

\section{Study objective 1: effects of digital primary care}

Evaluation of the impact of digital care on overall utilisation

Under the first objective, the study aims to investigate two possible effects of the use of digital primary care. The first effect relates to the question of whether digital primary care substitutes for traditional, in-office care or if the use of digital services leads to an overall increase in primary care utilisation. The guiding hypothesis is that digital care substitutes fully for traditional care and that there is no overall increase in the use of primary care services.

Given the current study design and context, the study aims to evaluate this issue by means of quasiexperimental methods, including matching and single difference $^{37} 38$ and by focusing on specific diagnosis-based episodes of care (Ashwood $e t a t^{28}$ address this question using matching and double difference. This approach is not possible in the current project as no preintervention data will be collected). The analysis will make use of a model that takes the data generating process into consideration, including the possible existence of a large share of zeros on the predictor side. Estimating the probability of using traditional primary care while controlling for the use of digital care will allow for the testing of the guiding hypothesis of full substitution of digital care. By generating a quasi-control group (through matching), the model can test whether the same holds also for the matched sample of patients.
Evaluation of digital primary care on prescription practices

The second effect of digital care under investigation concerns the question of whether there are any differences between the two models of primary care in antibiotic prescription behaviour. While all three index diagnoses are infections, a particular case may only require antibiotic treatment under certain conditions. While prudent and specific use of antibiotics is a general aim, the Swedish strategic programme against antibiotic resistance has developed guidance for the administration of antibiotics by diagnosis, also with respect to digital care, ${ }^{39}$ which will be used for defining the effects measured on prescription and use of antibiotics for each diagnostic group.

For each of the groups, the analytical aim is to estimate the probability of a correct prescription. Based on diagnose, available laboratory test results and patient information (age, gender) in the project database, an indicator variable will be generated taking the value 1 if correct prescription and 0 otherwise. The probability of correct prescription will be estimated by a logit regression model with a binary dependent variable estimating the effect of traditional and digital primary care. The general estimation model is:

$$
\mathrm{E}\left(\mathrm{Y} \mid \mathrm{X}, \mathrm{C}_{1}, \ldots, \mathrm{C}_{\mathrm{k}}\right)=\mathrm{P}\left(\mathrm{Y}=1 \mid \mathrm{X}, \mathrm{C}_{1}, \ldots . \mathrm{C}_{\mathrm{k}}\right)
$$

where $\mathrm{Y}$ is the dependent indicator variable of correct antibiotic prescription. $\mathrm{X}$ is a binary indicator variable for digital care $(\mathrm{X}=1 ; 0$ otherwise). $\mathrm{C}$ are control variables, including age, sex, income, education level and indicators of comorbidity.

\section{Adherence to prescribed antibiotic medicine}

Rational use of drugs, including antibiotics, in primary care is dependent on many factors beyond the formal prescription. ${ }^{40}{ }^{41}$ An important aspect is the patient's adherence to prescription. In the context of traditional and digital care, this may be of interest, as adherence is dependent on, for example, verbal instructions in the doctor-patient meeting and proximity of a pharmacy. The study will therefore estimate differences in dispensation between the two forms of providers with a similar model to (1), where $(\mathrm{Y})$ takes the value 1 if prescribed antibiotics are dispensed and 0 otherwise.

\section{Study objective 2: cost differences between forms of primary care provision}

Under the second objective, the study aims to compare the cost of care between the two forms of primary care, separately for each of the three index diagnoses. Furthermore, the costing analysis will estimate the resources used for both a single visit and for an entire episode of care.

\section{Estimating the cost of a digital contact and a traditional primary care visit}

The study will adopt a societal perspective by collecting cost estimates across all relevant sectors and the individual patient. The costing analysis will apply the general approach to costing healthcare programmes as described 
in, for example, Drummond et al. ${ }^{42}$ The approach involves the three main steps of identification (of all cost items), quantification (or measurement of resources) and valuation (of the items). Statistical analysis of cost differences will include the bootstrap approach.

The study will identify all cost units of both providers, patients, and any other part of society. Direct costs for providers include staff time and laboratory tests. Staff time will be limited to time spent by the treating physician. The indirect provider costs include administration, management, office rent and service development costs.

Patient direct cost items include user fees, pharmaceuticals and expenses for travel. The indirect cost carried by the patient is the value of time spent. This includes travel time, waiting time and the actual meeting with professional staff. Only the estimated time patients wait in the facility is included, as this time cannot be spent on productive work, that is, there is no clearly identifiable opportunity cost. For a digital visit, this means time spent with the digital device registering and waiting for a consultation. In traditional care, it is the time from arrival in the facility to the commencement of the consultation. Costs in the form of informal care by people related to the patient is assumed to be negligible for the studied diagnoses. Similarly, intangible costs such as pain and discomfort of care are assumed to the marginal and estimated to zero.

A large and decisive part of provider cost in healthcare is staff time. For digital services, time spent per patient as recorded by each providers' digital system will be used for calculating the average time spent per patient for the respective diagnosis. For traditional primary care, a survey of time spent per patient will be conducted among a sample of clinics. The questionnaire will separate patient time and time spent on patient-related administration.

Indirect provider costs (administration, management, office rent, development) for both forms of provision will be estimated with a top-down approach using costs from annual reports by private providers. To estimate development costs, which include staff training and software development, conventional accounting rules for immaterial assets write-off will be applied.

All patient time will be valued by the average Swedish gross salary plus social security contributions and benefits, for all patients aged 18-66. The study will apply the friction cost method for measuring indirect costs related to time spent by patients, by adjusting the salary level for labour market participation rate. ${ }^{43}$

\section{Estimating the cost of an episode of care}

The result of the above cost estimates will describe the difference between the two models of care for a single visit or digital contact. However, additional medical care may be needed if the treatment is ineffective. For management of chronic conditions, eHealth solutions have been shown to be effective in reducing health service consumption. ${ }^{44}$ To assess the differences in cost per case between the two forms of care, the study defines the episode of care for each specific condition under study. Each episode of care starts with a new contact, called an index visit, generating one of the three diagnoses LUTI, URTI or SSTI. The study further defines the episode as all visits to healthcare providers over a 2-week timeframe starting with the index date, similar to Gordon $e t a l .{ }^{26} \mathrm{~A}$ sensitivity analysis will test different timeframes, as factors such as waiting time and drug prescription can influence the length of an episode.

For visits to specialised outpatient clinics, the official reimbursement rate for the respective services will be applied. For the few hospital services that can be anticipated in these episodes, official average cost-per-patient data will be applied, representing the average cost per case in Swedish hospitals.

\section{Study objective 3: equality in health service utilisation}

In addition to effectiveness and costs, an important performance aspect of developed primary care is how utilisation is distributed across the population. The aim of the third study objective is to answer if there is a difference in socioeconomic distribution between digital and traditional primary care utilisation. The study will compare the distribution of utilisation by income and education levels across the two populations using one or the other form of service.

\section{Measuring inequalities}

The possible differences in utilisation will be analysed in three steps. The first is descriptive statistics of both forms of primary care utilisation across demographic and socioeconomic groups. Income is measured as individual total labour income and for education the International Standard Classification of Education is applied to define the levels of education status, as provided by Statistics Sweden.

In the second step, the study will calculate a concentration index (CI) as a summary measure of differences in the distribution of utilisation between the two forms of care. The method builds an index value from the cumulative distribution of healthcare utilisation and socioeconomic indicators ${ }^{19}$ and will produce comparable numbers of how consumption of services are distributed across users of the two models of care, by income and education. It runs between -1 and 1 and equals 0 when there is perfect equality, meaning the cumulative utilisation equals the distribution of the socioeconomic indicator. For the income distribution, the type of service with the highest (lowest) index number has the most prorich (propoor) distribution of utilisation. In the case of perfect inequality, it would take the extreme values if all healthcare was used by the least wealthy $(-1)$ or the wealthiest (1) individual. This can also be illustrated graphically by a concentration curve, which in the case of an index value 0 is a straight diagonal line. The estimation is defined as two times the covariance of the number of visits to either form of primary care $(\mathrm{u})$ and the relative fractional rank of the ith individual in the income distribution (R), divided by the mean of $\mathrm{u}(\mu)$ : 


$$
\mathrm{CI}=2 \operatorname{cov}\left(\mathrm{u}_{\mathrm{i}}, \mathrm{R}_{\mathrm{i}}\right) / \mu
$$

However, groups with lower socioeconomic status tend to have lower health status. ${ }^{45}$ When this is the case, differences in use of services will underestimate the inequalities. For the comparison of digital and traditional primary care, the above indices will ignore any differences in the composition of the two population groups using one or the other form of care. For this purpose, the third step will assess the distribution of utilisation related to households' socioeconomic status, after adjusting for differences in need across the population.

This is done by applying a horizontal equity approach. ${ }^{19}$ Horizontal equity occurs when individuals in equal need (as defined by selected indicators) are treated the same (in terms of utilisation), irrespective of income. The approach starts by obtaining an indirect standardisation of utilisation for healthcare need. An ordinary least squares regression estimates a predicted number of visits for each individual based on the variables age, sex, a morbidity index, labour market participation and ethnic background. The study will make use of the individual diagnosis information in the project database to create a disease burden index, using the variables for chronic illnesses and presence of other diagnoses, and test an alternative method with a vector of binary variables for presence of each diagnosis, similar to Gerdtham. ${ }^{46}$

The regression output provides the expected number of visits to the two forms of care the patient would use if she consumed care as a patient with the same characteristics do on average. The indirectly standardised utilisation for each individual is obtained as the difference between actual and predicted utilisation plus the sample mean utilisation. Then, a new needs-standardised CIs is calculated, which compares the equality in utilisation between the two forms of primary care services, adjusted for factors included in the regression. ${ }^{19}$

\section{DISCUSSION}

The current research project aims to contribute to the evidence on the effects, costs and distributional impacts of scaling up digital primary care in the context of a universal public healthcare system. The proposed analyses described in this protocol take a quantitative approach to evaluating the effects of digital care. Using a large database on individual patients and their characteristics, a number of specific questions will be addressed. However, there are also a number of limitations to the suggested approach. First, the absence of random allocation of patients to either of the two models of care will affect the strength of any conclusions about causal effects. The use of quasi-experimental methods to the data will offset some of these limitations.

Second, the quantitative approach will not be able to address other important questions of digital care, such as medical staff and patient perceptions and experiences of the care. Hence, the studies produced under this research project will complement those of other approaches. Third, the studies will not be able to differentiate between different forms of digital primary care. For example, it will not address the issue of whether synchronous consultations are more effective than asynchronous ones, or vice versa. Similarly, the research project aims to compare two distinct forms of primary care, not a situation where the models act in some combined or integrated form of service provision. Fourth, while the study will make use of a large database covering services over 2 years from a representative sample of regions, the application of digital primary care continuous to develop, both in its scope and form. As the technology and practice of digital primary care matures, it is likely that both patients and providers will adjust to its limits and possibilities. Obtaining an early understanding of these limitations and opportunities is critical for effective policy development, with relevant lessons for most other countries.

Finally, it is important to note that the specific issues of digital primary care and the methodologies applied for analyses which the research project aims to investigate are also relevant for any new form of primary care provision, for example, pharmacies and other retail clinics providing services traditionally conducted by in-office primary care. ${ }^{67}$ The current studies will thus be able to contribute to an improved understanding of the effects of changing primary care more broadly.

\section{Ethics and dissemination}

Ethical approval has been provided by the Swedish Ethical Review Authority (reference number 2019-01500). This is a registry-based study. As such, use of data follows the Swedish legislation and practice with regards to consent. For more information about ethical aspects and consent in registrybased studies in Nordic countries, see Ludvigsson $e^{a l .}$. $^{48}$

The outputs of this study are relevant to a wide set of stakeholders and asked for in many different fora. The results will therefore be disseminated both to the research community, healthcare decision-makers and to the general public. Each separate study objective as described above will generate at least one scientific article. The research team will also organise policy dialogues on digital primary care where evidence from the project will be presented.

\section{Author affiliations}

${ }^{1}$ Department of Clinical Sciences, Malmö, Lunds University Faculty of Medicine, Lund, Sweden

${ }^{2}$ Department of Medicine and Optometry, Linnaeus University Faculty of Health Social Work and Behavioural Sciences, Kalmar, Sweden

${ }^{3}$ Research and Development unit for Primary Care, Futurum Academy of Health and Care, Jonkoping, Sweden

${ }^{4}$ Department of Health, Medicine and Caring, Linköping University, Linkoping, Sweden

${ }^{5}$ Centre for Mathematical Sciences, Lund University Faculty of Engineering, Lund, Sweden

Contributors BE led the development of the study protocol. JW contributed to the design and conceptualisation of the protocol. JW and BE wrote the initial drafts of the protocol. HT and EA contributed to the identification and conceptualisation of the issues around primary care practices and processes. AL contributed to the 
statistical conceptualisation of the studies. All authors read and approved the final version of the manuscript.

Funding The project is partly funded by the Swedish Research Council for Health, Working Life and Welfare, grant number 2018-00093. This means the general design of the study was included in the funding proposal, but not the specific statistical methods. Additional resources for the study represent a minor part of funding and are not raised externally.

Competing interests None declared.

Patient and public involvement Patients and/or the public were not involved in the design, or conduct, or reporting, or dissemination plans of this research.

Patient consent for publication Not required.

Provenance and peer review Not commissioned; externally peer reviewed.

Open access This is an open access article distributed in accordance with the Creative Commons Attribution Non Commercial (CC BY-NC 4.0) license, which permits others to distribute, remix, adapt, build upon this work non-commercially, and license their derivative works on different terms, provided the original work is properly cited, appropriate credit is given, any changes made indicated, and the use is non-commercial. See: http://creativecommons.org/licenses/by-nc/4.0/.

\section{ORCID iD}

Jens Wilkens http://orcid.org/0000-0002-5336-8453

\section{REFERENCES}

1 OECD/EU. Health at a glance: Europe 2018: state of health in the EU cycle. Paris: OECD and the Europen Union, 2018.

2 Starfield B, Shi L, Macinko J. Contribution of primary care to health systems and health. Milbank Q 2005;83:457-502.

3 World Health Organization. World Health Report 2010: Health systems financing - the path to universal coverage. Geneva: World Health Organization, 2010.

4 WHO. From innovation to implementation: eHealth in the who European region. Copenhagen: WHO Regional Office for Europe, 2016.

5 OECD. Bringing health care to the patient: an overview of the use of telemedicine in OECD countries, in OECD health working paper. Paris: OECD, 2020.

6 Nakagawa K, Kvedar J, Yellowlees P. Retail outlets using telehealth pose significant policy questions for health care. Health Aff 2018;37:2069-75.

7 Wachter R. The digital doctor: hope, hype, and harm at the dawn of medicine's computer age. New York: McGraw-Hill, 2017.

8 Ekman B, Thulesius $\mathrm{H}$, Wilkens J, et al. Utilization of digital primary care in Sweden: descriptive analysis of claims data on demographics, socioeconomics, and diagnoses. Int J Med Inform 2019;127:134-40.

9 Statens Offentliga Utredningar. Digifysiskt vårdval. Tillgänglig primärvård baserad på behov och kontinuitet. Stockholm: Statens Offentliga Utredningar, 2019.

10 Anell A, Glenngård AH, Merkur S. Sweden - Health system review, in Health Systems in Transition. In: Merkur S, ed. The European Observatory on health systems and policies. Copenhagen, 2012.

11 Nya patientavgifter, 2019. Available: https://skr.se/tjanster/press/ nyheter/nyhetsarkiv/nyapatientavgifter2019.26198.html [Accessed 13 Feb 2020].

12 Anell A. Choice and privatisation in Swedish primary care. Health Econ Policy Law 2011;6:549-69.

13 Socialstyrelsen. Tillgänglighet I hälso- och sjukvården (in Swedish). Stockholm: Socialstyrelsen (The National Board of Health), 2018.

14 Doty MM, Tikkanen R, Shah A, et al. Primary care physicians' role in coordinating medical and health-related social needs in eleven countries. Health Aff 2020;39:115-23.

15 IVO. Digitala vårdtjänster. In: laktagelser $i$ korthet. Health and Social Care Inspectorate (IVO), 2019.

16 Ekman B. Cost analysis of a digital health care model in Sweden. Pharmacoecon Open 2018;2:347-54.

17 Ellegård LM, Kjellsson G. Telemedicine services associated with higher use of regular primary care in a Swedish region. 116. Lakartidningen, 2019.

18 OECD. Health for everyone?: social inequalities in health and health systems, OECD health policy studies. Paris: OECD Publishing, 2019.

19 van Doorslaer E, Koolman X, Jones AM. Explaining incomerelated inequalities in doctor utilisation in Europe. Health Econ 2004;13:629-47.
20 van Doorslaer $\mathrm{E}$, Wagstaff $\mathrm{A}$, van der Burg $\mathrm{H}$, et al. Equity in the delivery of health care in Europe and the US. J Health Econ 2000;19:19:553-83.

21 Salisbury C. Evaluation of Babylon GP at hand. Ipsos MORI York Health Economics Consortium, 2019.

22 Greenhalgh T, Shaw S, Wherton J, et al. Real-World implementation of video outpatient consultations at macro, meso, and micro levels: Mixed-Method study. J Med Internet Res 2018;20:e150.

23 Shi Z, Mehrotra A, Gidengil CA, et al. Quality of care for acute respiratory infections during direct-to-consumer telemedicine visits for adults. Health Aff 2018;37:2014-23.

24 Ray KN, Shi Z, Gidengil CA, et al. Antibiotic prescribing during pediatric direct-to-consumer telemedicine visits. Pediatrics 2019;143:e20182491.

25 Dullet NW, Geraghty EM, Kaufman T, et al. Impact of a UniversityBased outpatient telemedicine program on time savings, travel costs, and environmental pollutants. Value Health 2017;20:542-6.

26 Gordon AS, Adamson WC, DeVries AR. Virtual visits for acute, Nonurgent care: a claims analysis of Episode-Level utilization. J Med Internet Res 2017;19:e35.

27 Mehrotra A, Paone S, Martich GD, et al. A comparison of care at E-visits and physician office visits for sinusitis and urinary tract infection. JAMA Intern Med 2013;173:72.

28 Ashwood JS, Mehrotra A, Cowling D, et al. Direct-To-Consumer telehealth may increase access to care but does not decrease spending. Health Aff 2017;36:485-91.

29 Bavafa H, Hitt L, Terwiesch C. The impact of e-Visits in primary care: evidence on visit frequencies and patient health. 46. Wisconsin: Wisconsin School of Business, University of Wisconsin, 2016.

30 Armfield NR, Gray LC, Smith AC. Clinical use of Skype: a review of the evidence base. J Telemed Telecare 2012;18:125-7.

31 Armfield NR, Bradford M, Bradford NK. The clinical use of Skype-For which patients, with which problems and in which settings? A snapshot review of the literature. Int J Med Inform 2015;84:737-42.

32 Elbert NJ, van Os-Medendorp $\mathrm{H}$, van Renselaar W, et al. Effectiveness and cost-effectiveness of eHealth interventions in somatic diseases: a systematic review of systematic reviews and meta-analyses. J Med Internet Res 2014;16:e110-204.

33 Wade VA, Karnon J, Elshaug AG, et al. A systematic review of economic analyses of telehealth services using real time video communication. BMC Health Serv Res 2010;10:233.

34 CAMTÖ. Medicinsk bedömning och diagnostik av nytillkomna symtom via digitala vårdbesök. HTA-enheten: Örebro, 2018.

35 Bashshur RL, Howell JD, Krupinski EA, et al. The empirical foundations of telemedicine interventions in primary care. Telemed $J$ E Health 2016;22:342-75.

36 de la Torre-Díez I, López-Coronado M, Vaca C, et al. Cost-Utility and cost-effectiveness studies of telemedicine, electronic, and mobile health systems in the literature: a systematic review. Telemed J E Health 2015;21:81-5.

37 Wooldridge JM. Econometric analysis of cross section and panel data. Cambridge, MA and London, UK: The MIT Press, 2002.

38 Ravallion M. Evaluating Anti-Poverty programs, in policy research working paper. Washington, DC: The World Bank, 2005.

39 STRAMA. Rekommendationer för kvalitetsindikatorer vid digitala vårdmöten, 2017.

40 Fernandes M, Leite A, Basto M, et al. Non-adherence to antibiotic therapy in patients visiting community pharmacies. Int J Clin Pharm 2014;36:86-91.

41 Wickström Östervall L. Nudging to prudence? The effect of reminders on antibiotics prescriptions. J Econ Behav Organ 2017;135:39-52.

42 Drummond MF. Drummond MF, Sculpher MJ, Claxton K, et al, eds. Methods for the economic evaluation of health care programmes. 4th edn. Oxford University Press, 2015.

43 Koopmanschap MA, Rutten FF, van Ineveld BM, et al. The friction cost method for measuring indirect costs of disease. $J$ Health Econ 1995;14:171-89.

44 Odeh B, Kayyali R, Nabhani-Gebara S, et al. Evaluation of a telehealth service for COPD and HF patients: clinical outcome and patients' perceptions. J Telemed Telecare 2015;21:292-7.

45 Marmot M. Social determinants of health inequalities. Lancet 2005;365:1099-104.

46 Gerdtham UG, Sundberg G. Equity in the delivery of health care in Sweden. Scand J Soc Med 1998;26:259.

47 Ashwood JS, Gaynor M, Setodji CM, et al. Retail clinic visits for low-acuity conditions increase utilization and spending. Health Aff 2016;35:449-55

48 Ludvigsson JF, Håberg SE, Knudsen GP, et al. Ethical aspects of registry-based research in the Nordic countries. Clin Epidemiol 2015;7:491-508. 